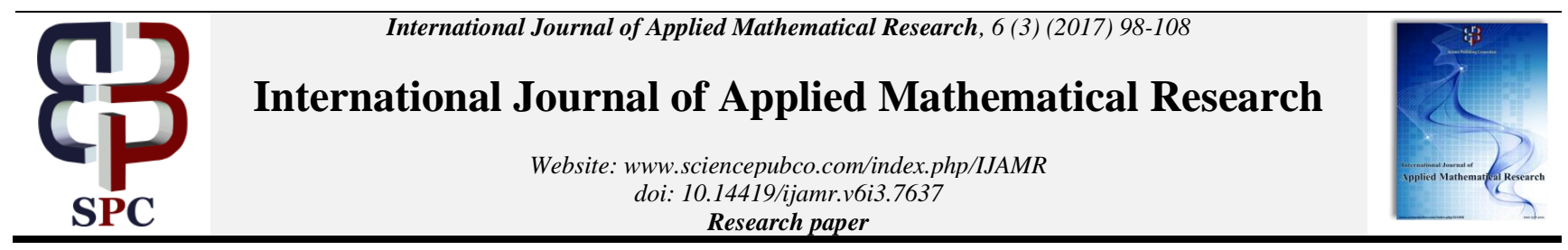

\title{
Norm of nonnegative and positive matrices
}

\author{
Alaa Mohammad Saeed Abu Alruz* \\ Financial \& Banking Sciences Department, Al-Balqaa Applied University, P.O.Box 107, Amman 11118 - Jordan \\ *Corresponding author E-mail:alaaruz@gmail.com
}

\begin{abstract}
The spectral radius $\mathrm{r}(\mathrm{A})$ of matrix $\mathrm{A}$ is the maximum modulus of the Eigen values. In this paper, the studies about the lower and upper bounds for the spectral radius and the lower bounds for the minimum eigen value of appositive and nonnegative matrices are investigate. The matrix norm, the spectral radius norm,and the column (row) sums of nonnegative and positive matrices are widely used to establish some inequalities for matrices. Then several existing results are improved for these inequalities for nonnegative and positive matrix. Furthermore, the lower and upper bounds of the Perron roots for nonnegative matrices are examined, and some upper bounds are computed.
\end{abstract}

Keywords: Nonnegative Matrix; Positive Matrix; Spectral Radius;Perron Roots of Nonnegative Matrix.

\section{Introduction}

The spectral radius function is one of the most important functions of matrices. It is closely related to matrix norms and the numerical radius.

Many functionals in matrix analysis are submultiplicative with respect to ordinary matrix multiplication, but the spectral radius is not. However, for nonnegative or positive semidefinite matrices $\mathrm{A}, \mathrm{B} \in \mathrm{Mn}$, the norm ( spectral radius ) of nonnegative and positive matrices is submultiplicative with respect to the nonnegative theorems:

$$
\min _{1 \leq i \leq n} \frac{1}{x_{i}} \sum_{j=1}^{n} a_{i j} x_{j} \leq r(A) \leq \max _{1 \leq i \leq n} \frac{1}{x_{i}} \sum_{j=1}^{n} a_{i j} x_{j}
$$

And

$$
\min _{1 \leq j \leq n} x_{j} \sum_{i=1}^{n} \frac{a_{i j}}{x_{i}} \leq r(A) \leq \max _{1 \leq j \leq n} x_{j} \sum_{i=1}^{n} \frac{a_{i j}}{x_{i}}
$$

(See Theorem 5.1.8 and 5.1.9).

This result, among other interesting properties of the spectral radius and the nonnegative and positive matrix, can be found in the famous book of Halmos [5] and that of Horn and Johnson [6]. Equalities and inequalities for the spectral radii of nonnegative and positive of matrices have been given by Zhang [15], Cheng, G-H., [3], Barra and Boumazgour [1].

The material of this research has been arranged, spread out in three parts. The arrangement of the subject matter is given in such a way to give a brief survey of results related to the norm of nonnegative and positive matrix.

Firstly, the study introduces some preliminary results in matrix theory that will be very useful in this research. These include some elements of the spectral theory, positive definite matrices, nonnegative and positive matrices, and matrix norm.

Secondly, the study deals with matrix norms and introduces the concept of spectral radius. Special emphasis is given to properties of the spectral radius, and presents several inequalities for the spectral radii of sums, products, and commutators of matrices.

Finally, the study introduces some basic definitions and properties of nonnegative matrices and positive matrices. Also it gives and proves some bounds for the Upper bounds of the Perron roots of nonnegative matrices.

\section{Previous studies}

1) The study of (PattrawutChansangiam_, PatcharinHemchote, PraiboonPantaragphong, 2009) aimed to develop inequalities for Kronecker products and Hadamard products of positive definite matrices. A number of inequalities involving powers, Kronecker powers, and Hadamard powers of linear combination of matrices are presented. In particular, $\mathrm{H}^{*}$ older inequalities and arithmetic mean-geometric mean inequalities for Kronecker products and Hadamard products are obtained as special cases.

2) The study of (Roger A. Horn and Fuzhen Zhang, 2010) aimed to prove Zhan's conjecture (the spectral radius of the Hadamard product of two square nonnegative matrices is not greater than the spectral radius of their ordinary product), and a related inequality for positive semidefinite matrices, using standard facts about principal sub matrices, Kronecker products, and the spectral radius.

3) The study of (Dongjun Chen and Yun Zhang, 2015) presented some spectral radius inequalities for nonnegative matrices. Used the ideas of Audenaert, and then proved the inequality which may be regarded as a Cauchy--Schwarz inequality for spectral radius of nonnegative matrices

$$
r\left(A^{\circ} B\right) \leq\left[r\left(A^{\circ} A\right)\right]^{1 / 2}\left[r\left(B^{\circ} B\right)\right]^{1 / 2}
$$

In addition, new proofs of some related results due to Horn and Zhang, Huang were also given. Finally, it interpolated Huang's inequality by proving 
$r\left(A_{1}{ }^{\circ} A_{2}{ }^{\circ} \ldots{ }^{\circ} A_{k}\right) \leq\left[r\left(A_{1} A_{2} \ldots A_{k}\right]^{1-\frac{2}{k}}\left[r\left(\left(A_{1}{ }^{\circ} A_{1}\right) \ldots\left(A_{k}{ }^{\circ} A_{k}\right)\right]^{\frac{1}{k}} \leq\right.\right.$ $r\left(A_{1} A_{2} \ldots A_{K}\right)$.

On the spectral radius of Hadamard products of nonnegative matrices

4) The study of (Koenraad M.R., 2010) aimed to prove an inequality for the spectral radius of products of non-negative matrices conjectured by Zhan. And showed that for all $n \times n$ non-negative matrices $A$ and $B, r\left(A^{\circ} B\right) \leq r\left[\left(A^{\circ} A\right)\left(B^{\circ} B\right)\right]^{\frac{1}{2}} \leq$ $r(A B)$, in which ${ }^{\circ}$ represents the Hadamard product.

5) The study of (Maozhong Fang, 2007) aimed to prove an upper bound for the spectral radius of the Hadamard product of nonnegative matrices and a lower bound for the minimum eigenvalue of the Fan product of M-matrices.

6) The study of (M. Goldberg, G. Zwas, 1974) characterized all nxn matrices whose spectral radius equals their spectral norm. it showed that for $n \geqslant 3$ the class of these matrices contains the normal matrices as a subclass.

7) The study of (Zejun Huang, 2010) aimed to prove the spectral radius inequality $r\left(A_{1}^{\circ} A_{2}^{\circ} \ldots{ }^{\circ} A_{k}\right) \leq r\left(A_{1} A_{2} \ldots A_{k}\right)$ for nonnegative matrices using the ideas of Horn and Zhang. It obtained the inequality $\left\|\mathrm{A}^{\circ} \mathrm{B}\right\| \leq \mathrm{r}\left(\mathrm{A}^{\mathrm{T}} \mathrm{B}\right)$ for nonnegative matrices, which improves Schur's classical inequality $\left\|\mathrm{A}^{\circ} \mathrm{B}\right\| \leq\|\mathrm{A}\|\|\mathrm{B}\|$, where $\|$.$\| denotes the spectral norm. It$ also gave counterexamples to two conjectures about the Hadamard product.

\section{Fundamentals of matrix analysis}

\subsection{Basic results in matrix theory}

Let $\mathrm{M}_{\mathrm{mn}}$ denote the space of all $\mathrm{m} \times \mathrm{n}$ complex matrices and let $\mathrm{M}_{\mathrm{n}}$ denote the algebra of all $n \times n$ complex matrices.

Definition 3.1.1: Let $A \in M_{n}$, Then a complex number $\lambda$ is called an eigenvalue of $A$, if there exists a nonzero vector $x \in C^{n}$. Such that $A x=\lambda x$. Such a vector $x$ is called an eigenvector of A associated with $\lambda$.

Definition 3.1.2: If $A \in M n$, then $\operatorname{det}(\lambda I-A)=0$ is called the characteristic equation of $\mathrm{A}$, where det (.) is the determinant function. The polynomial $p(\lambda)=\operatorname{det}(\lambda I-A)$ is called the characteristic polynomial of $A$. the set of all eigenvalues of $A$ is called the spectrum of A, denoted by $\sigma(A)$.

Theorem 3.1.3: If $A \in M_{n}$ then $\lambda \in \sigma(A)$ is an eigenvalue of $A$ if and only if $\operatorname{det}(\lambda I-A)=0$.

Definition 3.1.4:Let $A=\left[a_{i j}\right] \in M_{n}$. Then

1) The trace of $\mathrm{A}$ is given by $\operatorname{tr} \mathrm{A}=\sum_{\mathrm{i}=1}^{\mathrm{n}} \mathrm{a}_{\mathrm{ii}}$.

2) The transpose of $A$ is given by $A^{t=}\left[a_{j i}\right]$ and $A^{*}=\left[\overline{a_{j 1}}\right]$ is called the adjoint of $\mathrm{A}$.

Theorem 3.1.5: For all A, B $\in M_{n}$

1) $\sigma(\mathrm{AB})=\sigma(\mathrm{BA})$

2) If $\sigma(A)=\left\{\lambda_{1}, \ldots, \lambda_{n}\right\}$, then $\operatorname{det}(A)=\prod_{j=1}^{n} \lambda_{j}$, and $\operatorname{tr}(\mathrm{A})=\sum_{\mathrm{j}=1}^{\mathrm{n}} \lambda_{\mathrm{j}}$.

3) $\sigma\left(A^{*}\right)=\{\bar{\lambda}: \lambda \in \sigma(\mathrm{A})\}$.

Theorem 3.1.6: Let $A, B \in M_{n}$, and let $\alpha \in C$. Then

1) $\operatorname{det} \mathrm{AB}=(\operatorname{det} \mathrm{A})(\operatorname{det} \mathrm{B})$

2) $\operatorname{det}(\alpha \mathrm{A})=\alpha^{\mathrm{n}} \operatorname{det} A$

3) $\Sigma\left(\mathrm{A}^{\mathrm{k}}\right)=(\sigma(\mathrm{A}))^{\mathrm{k}}=\left\{\lambda^{\mathrm{k}}: \lambda \in \sigma(\mathrm{A})\right\}$, where $\mathrm{k}$ is a natural number.
4) $\sigma\left(\mathrm{A}^{\mathrm{t}}\right)=\sigma(\mathrm{A})$.

5) for any matrix $\mathrm{A}$ with rank at most $1, \sigma(\mathrm{A})=\{\operatorname{trA}, 0\}$

Theorem 3.1.7: Let $A, B \in M_{n}$ a $\in C$. Then

1) $\operatorname{tr}(\mathrm{A}+\mathrm{B})=\operatorname{tr} \mathrm{A}+\operatorname{tr} \mathrm{B}$.

2) $\operatorname{tra} \mathrm{A}=a \operatorname{tr} \mathrm{A}$

3) $\operatorname{tr} \mathrm{AB}=\operatorname{tr} \mathrm{BA}$

4) $\operatorname{tr} 0=0, \operatorname{tr} I_{n}=n$, where $I_{n}$ is the identity matrix of order $n$.

Theorem3.1.8: Let A,B $\in M_{n}, \alpha \in C$. Then

1) $\left(\mathrm{A}^{*}\right)^{*}=\mathrm{A}$

2) $(\mathrm{A}+\mathrm{B})^{*}=\mathrm{A}^{*}+\mathrm{B}^{*}$

3) $\left(\alpha A^{*}\right)=\bar{\alpha} A^{*}$.

4) $(\mathrm{A} \mathrm{B})^{*}=\mathrm{B}^{*} \mathrm{~A}^{*}$

5) $\operatorname{det}\left(\mathrm{A}^{*}\right)=\overline{\operatorname{det}(\mathrm{A})}$

6) $\operatorname{tr} \mathrm{A}^{*}=\overline{\operatorname{trA}}$.

7) $\operatorname{tr}^{*} \mathrm{~A} \geq 0$

8) $\sigma\left(\mathrm{A}^{*}\right)=\overline{\sigma(\mathrm{A})}$

Definition 3.1.9: $A, B \in M_{n}$ are called similar if there exists invertible $S \in M_{n}$ such that

$\mathrm{B}=\mathrm{S}^{-1} \mathrm{AS}$ or $\mathrm{A}=\mathrm{SBS}^{-1}$

Theorem 3.1.10:Similar matrices have the same eigenvalues.

Corollary 3.1.11: Similar matrices have the same determinant and trace.

Theorem 3.1.12: (The spectral mapping theorem), Let $A \in M$. Then for every polynomial $f \sigma(f(A))=f(\sigma(A))$.

Theorem 3.1.13:(Cayely - Hamilton). Every matrix satisfies its characteristic Polynomial (i.e, if $A \in M_{n}$ and $p$ is the characteristic polynomial of $\mathrm{A}$, then $\mathrm{P}(\mathrm{A})=0$ ).

Remark 3.1.14: Let $A \in M_{n}$ and let $k \in C$. Then $\sigma(k A)=k \sigma(A)$

Definition 3.1.15: If $A \in M_{n}$, then

1) $\mathrm{A}$ is called Hermitian if $\mathrm{A}^{*}=\mathrm{A}$.

2) $\mathrm{A}$ is called skew-Hermitian if $\mathrm{A}^{*}=-\mathrm{A}$.

3) $\mathrm{A}$ is called unitary if $\mathrm{A}^{*} \mathrm{~A}=\mathrm{AA}^{*}=\mathrm{I}$.

4) $\mathrm{A}$ is callednormal if $\mathrm{A}^{*} \mathrm{~A}=\mathrm{AA}^{*}$

It is obvious that Hermitian, skew-Hermitian and unitary matrices are normal matrices.

\section{Remark 3.1.16:}

1) The sum of two Hermitian matrices is Hermitian.

2) The product of two Hermitian matrices is Hermitian if and only if these matricescommute.

3) If $\mathrm{A} \in \mathrm{M}_{\mathrm{n}}$ then $\mathrm{AA}^{*}, \mathrm{~A}^{*} \mathrm{~A}, \mathrm{~A}+\mathrm{A}^{*}$ are Hermitian.

4) If $A \in M_{n}$ is Hermitian, then every eigenvalue of $A$ is a real number.

Remark 3.1.17: Let $A \in M_{n}$. Then

1) If $\mathrm{A}$ is unitary, then $|\operatorname{det} \mathrm{A}|=1$.

2) The product of two unitary matrices is unitary.

3) Ais unitary if and only if $A^{-1}=A^{*}$ is unitary.

4) If $\mathrm{A}$ is unitary, then every eigenvalue of $\mathrm{A}$ has modulus one.

Definition 3.1.18:If $\mathrm{x}=\left[\mathrm{x}_{1}, \mathrm{x}_{2}, \ldots, \mathrm{x}_{\mathrm{n}}\right]^{\mathrm{t}}, \mathrm{y}=\left[\mathrm{y}_{1}, \mathrm{y}_{2}, \ldots, \mathrm{y}_{\mathrm{n}}\right]^{t} \in \mathrm{C}^{\mathrm{n}}$, then the Euclidean inner product of xandyis given by: $(x, y)=\sum_{i=1}^{n} x_{i} \overline{y_{1}}$

Note that, $(\mathrm{x}, \mathrm{y})=\mathrm{y}^{*} \mathrm{x}$

Remark 3.1.19: Let $\mathrm{x}, \mathrm{y}, \mathrm{z} \in \mathrm{C}^{\mathrm{n}}, \alpha \in \mathrm{C}$ Then 
1) $(\mathrm{y}, \mathrm{x})=\overline{(\mathrm{x}, \mathrm{y})}$

2) $\quad(\alpha \mathrm{x}, \mathrm{y})=\alpha(\mathrm{x}, \mathrm{y})$

3) $(\mathrm{x}+\mathrm{y}, \mathrm{z})=(\mathrm{x}, \mathrm{z})+(\mathrm{y}, \mathrm{z})$

4) $(\mathrm{x}, \mathrm{x})=\sum\left|\mathrm{x}_{\mathrm{i}}\right|^{2} \geq 0$ with equality iff $\mathrm{x}=0$

5) $(\mathrm{x}, \alpha \mathrm{y})=\bar{\alpha}(\mathrm{x}, \mathrm{y})$

6) $(\mathrm{z}, \mathrm{x}+\mathrm{y})=(\mathrm{z}, \mathrm{x})+(\mathrm{z}, \mathrm{y})$

Definition 3.1.20: A matrix $B \in M_{n}$ is said to be unitarily equivalent to $A \in M_{n}$ if there is a unitary matrix $U \in M_{n}$ such that $\mathrm{B}=\mathrm{U}^{*} \mathrm{AU}$.

Theorem 3.1.21:(Schur's unitary triangularization theorem). Let $\mathrm{A} \in \mathrm{M}_{\mathrm{n}}$ with $\sigma(\mathrm{A})=\left\{\lambda_{1}, \lambda_{2}, \ldots, \lambda_{\mathrm{n}}\right\}$. Then there is a unitary matrix $\mathrm{U} \in \mathrm{M}_{\mathrm{n}}$ such that $\mathrm{U}^{*} \mathrm{AU}=\mathrm{T}$, where $\mathrm{T}=\left[\mathrm{t}_{\mathrm{ij}}\right] \in \mathrm{M}_{\mathrm{n}}$ is an upper triangular matrix with diagonal entries $t_{i i}=\lambda_{i}$ for $i=1,2 \ldots n$.

Theorem 3.1.22:(Spectral theorem for normal matrices). $A \in M_{n}$ is normal if and only if $\mathrm{A}$ is unitarily equivalent to a diagonal matrix (that is, $\mathrm{A}=\mathrm{UDU}^{*}$, where $\mathrm{D}$ is diagonal and $\mathrm{U}$ is unitary)

\subsection{Positive definite matrices}

Definition 3.2.1: A Hermitim matrix $A \in M_{n}$ is said to be positive definite, if $(A x, x)>0$ for all nonzero $x \in C^{n}$, and it is called positive semi-definite, if $(A x, x) \geq 0$ for all $x \in C^{n}$

\section{Remark 3.2.2:}

1) The sum of any two positive definite (semidefinite)matrices of the same size ispositive definite (semidefinite).

2) (The product of any two positive definite (semi-definite) matrices is positivedefinite (semi-definite) if and only if the two matrices commute.

3) Each eigenvalue of a positive definite (semi-definite) matrix is a positive) nonnegative) real number.

4) A Hermitian matrix whose eigenvalues are positive (nonnegative) is positivedefinite (positive semi-definite)

5) The trace and determinant of a positive definite (semidefinite) matrix are positive(nonnegative) real numbers.

Theorem 3.2.3: Let $A \in M_{n}$ be a positive semi-definite (definite) matrix and let $\mathrm{k} \geq 1 \mathrm{be}$ a given integer. Then there exists a unique positive semi-definite (definite) matrixB such that $A=B^{k}$, written as $B=A^{1 / k}$.

Theorem 3.2.4: $A$ matrix $A \in M_{n}$ is positive semi-definite if and only if $\mathrm{A}=\mathrm{BB}^{*}$ for some $\mathrm{B} \in \mathrm{M}_{\mathrm{n}}$. In the positive definite case $\mathrm{B}$ is taken to be invertible.

Definition 3.2.5: The eigenvalues of the matrix $\langle A\rangle=\left(A^{*} A\right)^{1 / 2}$ are called the singular values of $A$. They are denoted by $\mathrm{s}_{1}(\mathrm{~A})$ $\mathrm{s}_{2}(\mathrm{~A}) \ldots \mathrm{s}_{\mathrm{n}}(\mathrm{A})$ and they are arranged in non-increasing order so that $\mathrm{s}_{1}(\mathrm{~A}) \geq \mathrm{s}_{2}(\mathrm{~A}) \geq \ldots \geq \mathrm{s}_{\mathrm{n}}(\mathrm{A})$.

Theorem 3.2.6: (Singular value decomposition).If $A \in M_{n}$, then Amay be written in the form $\mathrm{A}=\mathrm{VDW}^{*}$, where $\mathrm{V}, \mathrm{W} \in \mathrm{M}_{\mathrm{n}}$ are unitary, and the matrix $\mathrm{D}=\operatorname{diag}\left(\mathrm{s}_{1}(\mathrm{~A}), \mathrm{s}_{2}(\mathrm{~A}), \ldots, \mathrm{s}_{\mathrm{n}}(\mathrm{A})\right.$.

Theorem 3.2.7:(Polar decomposition). If $A \in M_{n}$, then there exists a unitary matrix $\mathrm{U} \in \mathrm{M}_{\mathrm{n}}$ such that $\mathrm{A}=\mathrm{U}\langle\mathrm{A}\rangle$

Remark 3.2.8: Let $A \in M_{n}$ and let $U, V \in M_{n}$ be unitary, then

1) The matrices $\mathrm{A}^{*} \mathrm{~A}$ and $\mathrm{AA}^{*}$ are unitarily equivalent, and hence, they havethe same eigenvalues.

2) The matrices $\langle\mathrm{UAV}\rangle$ and (A) are unitarily equivalent, which implies that $s_{j}(U A V)=s_{j}(A)$ for all $j=1,2 \ldots n$.
3) If $\mathrm{A}$ is normal with eigenvalues $\lambda$ (A) ordered in such a way that $\left|\lambda_{1}(\mathrm{~A})\right| \geq \ldots \geq\left|\lambda_{\mathrm{n}}(\mathrm{A})\right|$, then $\mathrm{s}_{\mathrm{j}}(\mathrm{A})=\left|\lambda_{\mathrm{j}}(\mathrm{A})\right|$ for all $\mathrm{j}=$ $1,2, \ldots, \mathrm{n}$

Definition 3.2.9: A matrix $A=\left[a_{i j}\right] \in M_{n}$ is called diagonally dominant if $\left|a_{i i}\right|>\sum_{i \neq j}\left|a_{i j}\right|$ for $i=1,2 \ldots n$.

Definition 3.2.10: Let $A \in M$ mn For index sets $\alpha \in\{1,2, \ldots, m\}$ and $\beta \in\{1,2, . ., \mathrm{n}\}$, we denote the sub-matrix that lies in the rows of $\mathrm{A}$ indexed by $\alpha$ and the columns indexed by $\beta$ as $\mathrm{A}(\alpha, \beta)$. If $\mathrm{m}=$ $\mathrm{n}$ and $\beta=\alpha$, the submatrix $\mathrm{A}(\alpha, \alpha)$ is called a principal submatrix of $\mathrm{A}$ and is abbreviated $\mathrm{A}(\alpha)$.

Definition 3.2.11: Let $B=\left[b_{i j}\right] \in M_{n}$ and $A=\left[a_{i j}\right] \in M_{n}$. We write

$\mathrm{B} \geq 0$ if all $\mathrm{b}_{\mathrm{ij}} \geq 0$

$\mathrm{B}>0$ if all $b_{\mathrm{ijj}}>0$

$\mathrm{A} \geq \mathrm{B}$ if $\mathrm{A}-\mathrm{B} \geq 0$

$\mathrm{A}>\mathrm{B}$ if $\mathrm{A}-\mathrm{B}>0$.

If $\mathrm{A} \geq 0$, we say that $\mathrm{A}$ is a nonnegativematrix, and if $\mathrm{A}>0$, we say that $\mathrm{A}$ is a positive matrix. We define $|\mathrm{A}|=\left[\left|\mathrm{a}_{\mathrm{ij}}\right|\right]$.

Theorem 3.2.12:Let A, B, C, D $\in M_{n}$, Then

1) $|\mathrm{A}| \geq 0$ and $|\mathrm{A}|=0$ if and only if $\mathrm{A}=0$.

2) $|\mathrm{aA}|=|\mathrm{a} \| \mathrm{A}|$, for all complex numbers $\mathrm{a}$.

3) $|\mathrm{A}+\mathrm{B}| \leq|\mathrm{A}|+|\mathrm{B}|$.

4) If $\mathrm{A} \geq 0, \mathrm{~B} \geq 0$, and $\mathrm{a}, \mathrm{b} \geq 0$, then $\mathrm{aA}+\mathrm{bB} \geq 0$.

5) If $A \geq B$ and $C \geq D$, then $A+C \geq B+D$.

6) If $\mathrm{A} \geq \mathrm{B}$ and $\mathrm{B} \geq \mathrm{C}$, then $\mathrm{A} \geq \mathrm{C}$.

Theorem 3.2.13: Let $A, B, C, D \in M_{n}$, and let $x \in C^{n}$. Then.

1) $|\mathrm{Ax}| \leq|\mathrm{A}||\mathrm{x}|$

2) $|\mathrm{AB}| \leq|\mathrm{A}||\mathrm{B}|$.

3) $\left|\mathrm{A}^{\mathrm{m}}\right| \leq|\mathrm{A}| \mathrm{m}$ for all $\mathrm{m}=1,2, \ldots$

4) If $0 \leq \mathrm{A} \leq \mathrm{B}$ and $0 \leq \mathrm{C} \leq \mathrm{D}$, then $0 \leq \mathrm{AC} \leq \mathrm{BD}$.

5) If $0 \leq \mathrm{A} \leq \mathrm{B}$, then $0 \leq \mathrm{A}^{\mathrm{m}} \leq \mathrm{B}^{\mathrm{m}}$ for all $\mathrm{m}=1,2, \ldots$

6) If $A \geq 0$, then $A^{m} \geq 0$, and if $A>0$, then $A^{m}>0$ for all $m=$ $1,2, \ldots$

7) If $A>0, x \geq 0$, and $x \neq 0$, then $A x>0$.

8) If $\mathrm{A} \geq 0, \mathrm{x}>0$, and $\mathrm{Ax}=0$, then $\mathrm{A}=0$.

\subsection{Matrix norm}

Definition 3.3.1:Let $V$ be a vector space over a field F. A Function $\|\|:. \mathrm{V} \rightarrow \mathrm{R}$ is a vector norm if for all $\mathrm{x}, \mathrm{y} \in \mathrm{V}$.

1) $\|\mathrm{x}\| \geq 0$, and $\|\mathrm{x}\|=0$ if and only if $\mathrm{x}=0$ (Positivity).

2) $\|\mathrm{cx}\|=|\mathrm{c}|\|\mathrm{x}\|$ for all scalars $\mathrm{c} \in \mathrm{F}$ (Homogenity).

3) $\|x+y\| \leq\|x\|+\|y\|($ Triangle inequality).

For a vectorx $=\left(\mathrm{x}_{1}, \mathrm{x}_{2}, \ldots, \mathrm{x}_{\mathrm{n}}\right) \in \mathrm{C}^{\mathrm{n}}$, we define

$$
\begin{gathered}
\|x\|_{p}=\left(\sum_{j=1}^{n}\left|x_{j}\right|^{p}\right)^{\frac{1}{p}}, 1 \leq p<\infty \\
\|x\|_{\infty}=\lim _{p \rightarrow \infty}\|x\|_{p}=\max _{1 \leq j \leq n}\left|x_{j}\right| .
\end{gathered}
$$

For each $1 \leq \mathrm{p} \leq \infty,\|\mathrm{x}\|_{\mathrm{p}}$ defines a norm on $\mathrm{C}^{\mathrm{n}}$. These are called the $\mathrm{p}$-norms or $\ell_{\mathrm{p}}$ norms. While for $0<p<1$ this defines a quasi norm. Instead of the triangle inequality we have.

$$
\|\mathrm{x}+\mathrm{y}\|_{\mathrm{p}} \leq 2^{1 / \mathrm{p}-1}\left(\|\mathrm{x}\|_{\mathrm{p}}+\|\mathrm{y}\|_{\mathrm{p}}\right), 0<p<1
$$

Definition 3.3.2: A function $N: M \rightarrow R$ is a matrix norm if for all $A, B \in M_{n}$ it satisfies the following axioms: 
1) $\mathrm{N}(\mathrm{A}) \geq 0$ and $\mathrm{N}(\mathrm{A})=0$ if and only $\mathrm{A}=0$.

2) $\mathrm{N}(\mathrm{aA})=|\mathrm{a}| \mathrm{N}(\mathrm{A})$ for all complex numbers $\mathrm{a}$.

3) $N(A+B) \leq N(A)+N(B)$.

4) $\mathrm{N}(\mathrm{AB}) \leq \mathrm{N}(\mathrm{A}) \mathrm{N}(\mathrm{B})$.

Remark 3.3.3: A vector norm on $M_{n}$, that is a function that satisfies (1) - (3) and not necessarily (4), is often called a generalized matrix norm.

Examples 3.3.4: Let $=\left\lfloor a_{i j}\right\rfloor \in M_{n}$. Then

1) The $\ell_{1}$ norm is defined by $\|\mathrm{A}\|_{1}=\sum_{\mathrm{i}, \mathrm{j}=1}^{\mathrm{n}}\left|\mathrm{a}_{\mathrm{ij}}\right|$.

Note that $\|.\|_{1}$ is a matrix norm because

$$
\begin{aligned}
& \|A B\|_{1}=\sum_{i, j=1}^{n}\left|\sum_{k=1}^{n} a_{i k} b_{k j}\right| \leq \sum_{i, j, k=1}^{n}\left|a_{i k} b_{k j}\right| \\
& \leq \sum_{i, j, k, m=1}^{n}\left|a_{i k} b_{m j}\right|=\left(\sum_{i, k=1}^{n}\left|a_{i k}\right|\right)\left(\sum_{j, m=1}^{n}\left|b_{m j}\right|\right.
\end{aligned}
$$

$=\|\mathrm{A}\|_{1}\|\mathrm{~B}\|_{1}$

2) The $\ell_{2}$ norm (or the Euclidean norm) is defined by

$\|\mathrm{A}\|_{2}=\left(\sum_{\mathrm{i}, \mathrm{j}=1}^{\mathrm{n}}\left|\mathrm{a}_{\mathrm{ij}}\right|^{2}\right)^{\frac{1}{2}}$

Note that $\|.\|_{2}$ is a matrix norm because

$$
\begin{aligned}
& \|A B\|_{2}^{2}=\sum_{i, j=1}^{n}\left|\sum_{k=1}^{n} a_{i k} b_{k j}\right|^{2} \\
& \leq \sum_{i, j=1}^{n}\left(\sum_{k=1}^{n}\left|a_{i k}\right|^{2}\right)\left(\sum_{m=1}^{n}\left|b_{m j}\right|^{2}\right)
\end{aligned}
$$

$=\|\mathrm{A}\|_{2}^{2}\|\mathrm{~B}\|_{2}^{2}$

This inequality is just the Cauchy - Schwarz inequality. When applied to matrices, this norm is sometimes called the Forbenius norm, the Schur norm, or the Hilbert - Schmidt norm.

3) The maximum column sum matrix norm is defined by

$$
\||A|\|_{1}=\max _{1 \leq j \leq n} \sum_{i=1}^{n}\left|a_{i j}\right|
$$

4) The maximum row sum matrix norm is defined by

$\||A|\|_{\infty}=\max _{1 \leq i \leq n} \sum_{j=1}^{n}\left|a_{i j}\right|$

Note that, the norms $\||.|\|_{1}$, and $\||.|\|_{\infty}$ are induced by the $\ell_{1}$ and $\ell_{\infty}$ vectors norms, respectively, and hence must be matrix norms.

5) The spectral norm ( or the usual operator norm )is defined by

$$
\|\mathrm{A}\|=\max _{\|\mathrm{x}\|=1}\|\mathrm{Ax}\|
$$

Note that, for any matrix $A \in M_{n}$,

a) $\|\mathrm{A}\|=\max _{\|\mathrm{x}\|=\|\mathrm{y}\|=1}|(\mathrm{y}, \mathrm{Ax})|$.

b) If $A \in M_{n}$ is Hermitian, then, $\|A\|=\max _{\|x\|=1}|(x, A x)|$. c) If $A \in M_{n}$ is a unitary, then $\|A\|=1$.

d) $\left\|A^{\mathrm{k}}\right\| \leq\left\|A^{\mathrm{k}}\right\|$, for $\mathrm{k}=1,2, \ldots$

e) If $|A| \leq|B|$, then $\|A\|_{2} \leq\|B\|_{2}$.

6) The norm $\|\mathrm{A}\|_{\infty}=\max _{1 \leq \mathrm{i}, \mathrm{j} \leq \mathrm{n}}\left|\mathrm{a}_{\mathrm{ij}}\right|$ is a generalized matrix norm.

Definition 3.3.5: Let $\in M_{n}$. Then the numerical radius $w(A)$ of $A$ is defined by

$\mathrm{w}(\mathrm{A})=\max _{\|\mathrm{x}\|=1}|(\mathrm{Ax}, \mathrm{x})|$

Theorem3.3.6: The numerical radius $w($.$) is a norm on M_{n}$. That is, for all $A, B \in M_{n}$ and $a \in C$, we have

1) $w(A) \geq 0$ and $w(A)=0$ if and only if $A=0$.

2) $w(a A)=|a| w(A)$ for all complex numbers $a$.

3) $w(A+B) \leq w(A)+w(B)$.

Theorem 3.3.7: If $\in M_{n}$, then

(1) $w(A)=w\left(A^{*}\right)$.

(2) $w\left(U^{*} A U\right)=w(A)$ for every unitary $U \in M$.

(3) $w(A) \leq\|A\|$.

If $\mathrm{A}$ is normal, we have $\mathrm{w}(\mathrm{A})=\|\mathrm{A}\|$.

Theorem3.3.8: The norms $w($.$) and \|$.$\| are equivalent on M_{n}$. In fact for every $A \in M_{n}$, we have

$$
\mathrm{w}(\mathrm{A}) \leq\|\mathrm{A}\| \leq 2 \mathrm{w}(\mathrm{A}) .
$$

Proof: Note that,

$$
\mathrm{w}(\mathrm{A}) \leq\|\mathrm{A}\|
$$

Top prove that $\|\mathrm{A}\| \leq 2 \mathrm{w}(\mathrm{A})$, note that

$$
\|\mathrm{A}\|=\frac{1}{2}\left\|\left(\mathrm{~A}+\mathrm{A}^{*}\right)+\left(\mathrm{A}-\mathrm{A}^{*}\right)\right\|
$$

$\leq \frac{1}{2}\left\|\mathrm{~A}+\mathrm{A}^{*}\right\|+\frac{1}{2}\left\|\mathrm{~A}-\mathrm{A}^{*}\right\| \quad$ (By the triangle inequality)

$=\frac{1}{2} \mathrm{w}\left(\mathrm{A}+\mathrm{A}^{*}\right)+\frac{1}{2} \mathrm{w}\left(\mathrm{A}-\mathrm{A}^{*}\right) \quad($ by Theorem 1.3 .7$)$

(Since $\mathrm{A}+\mathrm{A}^{*}$ and $\mathrm{A}-\mathrm{A}^{*}$ are normal)

$$
\leq \mathrm{w}(\mathrm{A})+\mathrm{w}\left(\mathrm{A}^{*}\right)=2 \mathrm{w}(\mathrm{A}) \quad(\text { byTheorem1.3.7) }
$$

Remark 3.3.9: $w($.$) is not submultiplicative, that is it is not true$ that

$$
w(A B) \leq w(A) w(B)
$$

Even if $=$ BA. To see this,

$$
\text { Let } A=\left[\begin{array}{llll}
0 & 0 & 0 & 0 \\
1 & 0 & 0 & 0 \\
0 & 1 & 0 & 0 \\
0 & 0 & 1 & 0
\end{array}\right] \text { andB }=A^{2}
$$

Then

$$
\begin{gathered}
A B=B A=A^{3}, w(A)=\cos \frac{\pi}{5}=0.80901699 \\
w(B)=w\left(A^{2}\right)=w\left(A^{3}\right)=w(A B)=0.5
\end{gathered}
$$

Thus

$$
\mathrm{w}(\mathrm{AB})=0.5>w(\mathrm{~A}) \mathrm{w}(\mathrm{B})=0.4045085
$$


Remark 3.3.10: Let $A, B \in M_{n}$. Then

1) If $A, B$ are normal, then $w(A B) \leq w(A) w(B)$. To see this , note that

$$
w(A B) \leq\|A B\| \leq\|A\|\|B\|=w(A) w(B) .
$$

2) If $A, B$ are arbitrary, then $w(A B) \leq 4 w(A) w(B)$. Note that,

$$
\begin{aligned}
w(A B) \leq\|A B\|\|A\|\|B\| & \leq(2 w(A))(2 w(B)) \\
& =4 w(A) w(B)
\end{aligned}
$$

3) $w\left(A^{k}\right)$ for $k=1,2, \ldots$

For recent numerical radius inequalities, we refer to Kittaneh (2003 and 2005).

Definition 3.3.11:A matrix norm $\|$.$\| on M_{n}$ is said to be unitarily invariant if

$$
\|U A V\|=\|A\|
$$

For all $A \in M_{n}$ and for all unitary matrices, $V \in M_{n}$.

Theorem 3.3.12: The spectral norm is a unitarily invariant matrix norm.

Proof:

Let $A \in M_{n}$ and $U, V \in M_{n}$ be unitary, and let $B=U A V$. Then

$\|B\|=\|U A V\| \leq\|U\|\|A\|\|V\|=\|A\|$.

Since $A=U^{*} B V^{*}$ it follows that

$\|A\|=\left\|U^{*} B V^{*}\right\| \leq\left\|U^{*}\right\|\|B\|\left\|V^{*}\right\|=\|B\|$.

So

$\|A\|=\|B\|=\|U A V\|$.

Theorem3.3.13:The Euclidean norm is a unitarily invariant matrix norm.

\section{Proof:}

Let $A \in M_{n}$ and $U, V \in M_{n}$ be unitary, and let $B=U A V$. Then

$$
\begin{aligned}
& \quad\|B\|_{2}^{2}=\operatorname{tr} B^{*} B=\operatorname{tr}\left(V^{*} A^{*} U^{*} U A V\right)=\operatorname{tr}\left(V^{*} A^{*} A V\right) \\
& =\operatorname{tr} A^{*} A=\|A\|_{2}^{2}
\end{aligned}
$$

\section{Spectral radius inequalities}

\subsection{Properties of the spectral radius}

The following properties of the spectral radius can be found in horn and Johnson [6].

Definition 4.1.1: The spectral radius $r(A)$ of a matrix $A \in M_{n}$ is $r(A)=\max \{|\lambda|: \lambda$ is an eigenvalue of $A\}$.

Observe that if $\lambda$ is any eigenvalue of, then $|\lambda| \leq r(A)$.

Theorem 4.1.2: If $N($.$) is any matrix norm and if A \in M_{n}$, then $r(A) \leq N(A)$.

Proof:

Let $\lambda \in \sigma(A)$ such that $|\lambda|=r(A)$ and let $x \in C^{n}$ be a nonzero vector such that $A x=\lambda x$.

If $X=[x: x: \ldots: x]$, then $|\lambda| N(X)=N(\lambda X)=N(A X) \leq$ $N(A) N(X)$. Since $N(X) \neq 0$, we have $|\lambda| \leq N(A)$, and so $r(A) \leq$ $N(A)$.
Corollary 4.1.3: If $A \in M_{n}$, then $(A) \leq\|A\|$, and equality holds if $A$ is normal.

Remark 4.1.4: If $A, B \in M_{n}$, then $r(A B)=r(B A)$.

To see this, use the fact $\sigma(A B)=\sigma(B A)$.

Theorem 4.1.5: If $A, B \in M_{n}$ and $A B=B A$, Then

1) $r(A+B) \leq r(A)+r(B)$

2) $r(A B) \leq r(A) r(B)$.

Proof:

(1) Since $A B=B A$, by schur's theorem there is a unitary matrix $U \in M_{n}$ such that

$U^{*} A U$ And $U^{*} B U$ are both upper triangular, I.e,

$$
T_{1=} U^{*} A U=\left[\begin{array}{ccccc}
\lambda_{1} & a_{12} & a_{13} & \ldots . . & a_{1 n} \\
0 & \lambda_{2} & a_{23} & \ldots . . & a_{2 n} \\
0 & 0 & \lambda_{3} & \ldots . . & a_{3 n} \\
\vdots & \vdots & \vdots & \ddots & \vdots \\
0 & 0 & 0 & \ldots . . & \lambda_{n}
\end{array}\right]
$$

Where $\lambda_{i}, i=1,2, \ldots, n$ are the eivgenvalues of $A$, and

$$
T_{2}=U^{*} B U=\left[\begin{array}{ccccc}
\mu_{1} & b_{12} & b_{13} & \ldots & b \\
0 & \mu_{2} & b_{23} & \ldots & b_{2 n} \\
0 & 0 & \mu_{3} & \ldots & b_{3 n} \\
\vdots & \vdots & \vdots & \ddots & \vdots \\
0 & 0 & 0 & \ldots & \mu_{n}
\end{array}\right]
$$

Where $\mu_{i}, i=1,2, \ldots, n$ are the eivgenvalues of $\mathrm{B}$.

Note that $\sigma(A)=\sigma\left(T_{1}\right)=\left\{\lambda_{i}: i=1, \ldots, n\right\}$, and $\sigma(B)=$ $\sigma\left(T_{2}\right)=\left\{\mu_{I}: i=1, \ldots n\right\}$.

Now, $\sigma(\mathrm{A}+B) \subseteq \sigma(A)+\sigma(B)$, and $\sigma(A B) \subseteq \sigma(A) \sigma(B)$.

So, we have $r(A+B) \leq r(A)+r(B)$ and $r(A B) \leq r(A) r(B)$.

Remark 4.1.6:If $A, B \in M_{n}$ do not commute, then Theorem 4.1.5 is false. To see this consider the following example.

Let $A=\left[\begin{array}{ll}0 & 1 \\ 0 & 0\end{array}\right], B=\left[\begin{array}{ll}0 & 0 \\ 1 & 0\end{array}\right]$

Then $r(A)=0$ and $r(B)=0$, but $r(A+B)=1$.

So, $r(A+B)=1>r(A)+r(B)=0$.

Lemma 4.1.7: If $\in M_{n}$, then

$r(A)=\inf \left\{\left\|S^{-1} A S\right\|: S \in M^{n}\right.$ is invertible $\}$.

Proof:

If $S \in M_{n}$ invertible, then

$r(A)=r\left(S^{-1} A S\right) \leq\left\|S^{-1} A S\right\|$.

So,

$r(A) \leq \inf \left\{\left\|S^{-1} A S\right\|: S \in M^{n}\right.$ is invertible $\}$.

By the Schur traingularization theorem, there is a unitary matrix $U$ and an upper triangular matrix $\mathrm{T}$ with diagonal entries $\lambda_{1}, \ldots . \lambda_{n}$ (the eigenvalues of $A$ ), such that $U^{*} A U=T$

Set $D_{1}=\operatorname{diag}\left(t, t^{2}, t^{3}, \ldots t^{n}\right)$ for $t>0$, and comput $D_{t} T D_{t}^{-1}$. 
$D_{t} T D_{t}^{-1}=\left[\begin{array}{ccccc}\lambda_{1} & t^{-1} d_{12} & t^{-2} d_{13} & \ldots & t^{-n+1} d_{1 n} \\ 0 & \lambda_{2} & t^{-1} d_{23} & \ldots & t^{-n+2} d_{2 n} \\ 0 & 0 & \lambda_{3} & \ldots & t^{-n+3} d_{3 n} \\ \vdots & \vdots & \vdots & \ddots & \vdots \\ 0 & 0 & 0 & \ldots & \lambda_{n}\end{array}\right]$

Let $S_{1=} U D_{t}^{-1}$. Then $S_{t}^{-1} A S_{t}=D_{t} U^{*} A U D_{t}^{-1}=D_{t} T D_{t}^{-1}$

Thus, for $t>0$ large enough, we can be certain that the sum of all the absolute values of the off-diagonal entries of $S_{t}^{-1} \mathrm{~A} S_{t}$, can be made arbitrary small. It follows that

$\left\|S_{t}^{-1} A S_{t}-\operatorname{diag}\left(\lambda_{1}, \ldots, \lambda_{n}\right)\right\| \rightarrow 0$ as $t \rightarrow \infty$.

Thus

$$
\left\|S_{t}^{-1} A S_{t}\right\| \rightarrow\left\|\operatorname{diag}\left(\lambda_{1}, \ldots, \lambda_{n}\right)\right\|=\max _{1 \leq i \leq n}\left|\lambda_{i}\right|=r(A) .
$$

Now,

$r(A) \leq \inf \left\|S^{-1} A S\right\| \leq\left\|S_{t}^{-1} A S_{t}\right\|$, for all $t>0$.

Letting $t \rightarrow \infty$, we obtain

$r(A) \leq \inf \left\|S^{-1} A S\right\| \leq r(A)$, and so

$r(A)=\inf \left\|S^{-1} A S\right\|$.

Theorem 4.1.8: Let $\in M_{n}$. Then $\lim _{k \rightarrow \infty} A^{k}=0$, if and only if $r(A)<1$

Proof: If $A^{k} \rightarrow 0$ as $k \rightarrow \infty$, and if $x \neq 0$ is a vector such that

$A x=\lambda x$, Then

$A^{k} x=\lambda^{k} x \rightarrow 0$ Only if $|\lambda|<1$.since this inequality must hold for every eigenvalue of $\mathrm{A}$,We conclude that $r(A)<1$.

Conversely,

If $r(A)<1$, then by lemma 4.1.7

$\inf \left\{\left\|S^{-1} A S\right\|: S\right.$ is invertible $\}<1$

And so there is an invertible matrix $S \in M_{n}$ such that $\left\|S^{-1} A S\right\|<1$. But then

$\left\|\left(S^{-1} A S\right)^{k}\right\| \leq\left\|S^{-1} A S\right\|^{k} \rightarrow 0$ as $k \rightarrow \infty$.

Thus $\left(S^{-1} A S\right)^{k} \rightarrow 0$ as $k \rightarrow \infty$, which implies that $S^{-1} A S \rightarrow$ 0 as $k \rightarrow \infty$, and so

$$
A^{k} \rightarrow 0 \text { as } k \rightarrow \infty
$$

Corollary4.1.9: (Spectral radius formula). If $A \in M_{n}$, then $r(A)=$ $\lim _{k \rightarrow \infty}\left\|A^{k}\right\|^{1 / k}$.

\section{Nonnegative and positive matrices}

\subsection{Nonnegative matrices}

Theorem 5.1.1:Let $B \in M_{n}$. If $|A| \leq B$, Then $r(A) \leq r(|A|) \leq$ $r(B)$.

Proof: For every $\mathrm{m}=1,2 \ldots$ We have $\left|A^{m}\right| \leq|A|^{m} \leq B^{m}$

And

$\left\|A^{m}\right\|_{2} \leq\left\||A|^{m}\right\|_{2} \leq\left\|B^{m}\right\|_{2}$
Then

$\left\|A^{m}\right\|_{2}^{1 / m} \leq\left\||A|^{m}\right\|_{2}^{1 / m} \leq\left\|B^{m}\right\|_{2}^{1 / m}$ for all $\mathrm{m}=1,2 \ldots$

If we know let $\mathrm{m} \rightarrow \infty$ and apply Corollary 4.1.9, we have that $r(A) \leq r(|A|) \leq r(B)$.

Corollary5.1.2: Let , $B \in M_{n}$. If $0 \leq A \leq B$, then $r(|A|) \leq r(B)$.

Corollary 5.1.3: Let $A \in M_{n}$. If $A \geq 0$ and if $\tilde{A}$ is any principal submatrix of A, then $r(\tilde{A})=r(\breve{A})$

Proof:

Let $1 \leq \mathrm{r} \leq \mathrm{n}$ and let $\tilde{A}$ be an $\mathrm{r} \times \mathrm{r}$ principal square submatrix of $A$, let $\breve{A}$ denote the $\mathrm{n} \times \mathrm{n}$ matrix formed by placing the entries of $\tilde{A}$ in their former position and Placing 0's elsewhere. Then

$r(\tilde{A})=r(\breve{A})$ and $0 \leq \breve{A} \leq A$.

So,

$r(\tilde{A})=r(\breve{A})<r(\mathrm{~A}) \quad($ by corollary 5.1 .2$)$

Lemma 5.1.4:Let $A \in M_{n}$, and suppose that $A \geq 0$. If the row sums of $A$ are constant, then $\mathrm{r}(\mathrm{A})=\||A|\|_{\infty}$. If the column sums of $A$ are constant, then $r(A)=\||A|\|_{1}$

Proof: We know that $r(A) \leq\||A|\|$ for any matrix norm $\|||$.$\| .$ But if the row sums are constant, $\mathrm{x}=[1,1, \ldots, 1]^{t}$ is an eigenvector with eigenvalue $\||A|\|_{\infty}$, and so $r(A)=\||A|\|_{\infty}$. The other assertion follows by similar argument.

Theorem 5.1.5: Let $A \in M_{n}$, and suppose $A>0$. Then

$$
\min _{1 \leq i \leq n} \sum_{j=1}^{n} a_{i j} \leq r(A) \leq \max _{1 \leq i \leq n} \sum_{j=1}^{n} a_{i j}
$$

And

$$
\min _{1 \leq j \leq n} \sum_{i=1}^{n} a_{i j} \leq r(A) \leq \max _{1 \leq j \leq n} \sum_{i=1}^{n} a_{i j}
$$

Proof:

Let $\alpha=\min _{1 \leq i \leq n} \sum_{j=1}^{n} a_{i j}$, and let $B=\left|b_{i j}\right| \in M_{n}$ Such that $\mathrm{A} \geq \mathrm{B} \geq 0$, $\sum_{j=1}^{n} b_{i j}=\alpha$ for all,

$i=1,2, \ldots, n$ Then

$r(B)=\|B\|_{\infty}=\alpha$

And

$r(B)<r(A) \quad$ (By corollary 5.1.2)

Let $\beta=\max _{1 \leq i \leq n} \sum_{j=1}^{n} a_{i j}$, and let $\mathrm{C}=\left\lfloor c_{i j}\right\rfloor \in M_{n}$ such that $\mathrm{C} \geq \mathrm{A} \geq$ 0 And $\sum_{j=1}^{n} c_{i j}=\beta$ for all

$i=1,2, \ldots, n$.Then

$$
r(C)=\||C|\|_{\infty}=\beta
$$

And

$r(C) \geq r(A) \quad($ By corollary 5.1.2) 
The column sum bounds follow form applying the row sum bounds to $A^{1}$

Corollary 5.1.6: Let $A \in M_{n}$. If $A \geq 0$ and $\sum_{j=1}^{n} a_{i j}>0$ for all $i=1,2, \ldots, n$, then

$$
r(A)>0
$$

\section{Proof:}

Since $A \geq 0$ and $\sum_{j=1}^{n} a_{i j}>0$, we have

$$
\min _{1 \leq i \leq n} \sum_{j=1}^{n} a_{i j} \leq r(A) \quad \text { ( by Theorem 5.1.5) }
$$

So,

$r(A)>0$.

Corollary5.1.7: Let $A \in M_{n}$, and $A>0$. Then $r(A)>0$.

Theorem 5.1.8: Let $A \in M_{n}$, and suppose $A \geq 0$. Then for any positive vector $\mathrm{x} \in C^{n}$

We have

$$
\min _{1 \leq i \leq n} \frac{1}{x_{i}} \sum_{j=1}^{n} a_{i j} x_{j} \leq r(A) \leq \max _{1 \leq i \leq n} \frac{1}{x_{i}} \sum_{j=1}^{n} a_{i j} x_{j}
$$

And

$$
\min _{1 \leq j \leq n} x_{j} \sum_{i=1}^{n} \frac{a_{i j}}{x_{i}} \leq r(A) \leq \max _{1 \leq j \leq n} x_{j} \sum_{i=1}^{n} \frac{a_{i j}}{x_{i}}
$$

\section{Proof:}

Since $r\left(S^{-1} A S\right)=r(A)$ whenever $\mathrm{S}$ is invertible, if $\mathrm{S}=\operatorname{diag}\left(x_{1}, x_{2}, \ldots, x_{n}\right)$, all $x_{i}>0$, and since $A \geq 0$, then

$$
S^{-1} A S=\left\lfloor a_{i j} x_{j} x_{i}^{-1}\right\rfloor \geq 0 .
$$

So,

$$
\begin{aligned}
\min _{1 \leq i \leq n} \frac{1}{x_{i}} \sum_{j=1}^{n} a_{i j} x_{j} \leq & r\left(S^{-1} A S\right) \\
& \left.\leq \max _{1 \leq i \leq n} \frac{1}{x_{i}} \sum_{j=1}^{n} a_{i j} x_{j} \text { (by Theorem } 5.1 .5\right)
\end{aligned}
$$

Corollary 5.1.9: Let $A \in M_{n}$, let $x \in R^{n}$, and suppose that $A \geq$ 0 and $x>0$. If

$\alpha, \beta \geq 0$ are such that $\alpha x<A x<\beta x$, Then

$$
\alpha \leq r(A) \leq \beta
$$

If $\alpha x<A x$, then

$$
\begin{gathered}
\alpha<r(A) . \\
\text { If } A x<\beta x, \text { then } \\
r(A)<\beta
\end{gathered}
$$

\section{Proof:}

If $\alpha x \leq A x$, then $A x-\alpha x \geq 0$. So

$$
\sum_{j=1}^{n} a_{i j} x_{j}-\alpha x_{i} \geq 0, \text { for all } i=1,2, \ldots, n
$$

Then

$$
\alpha \leq \min _{1 \leq i \leq n} x_{i}^{-1} \sum_{j=1}^{n} a_{i j} x_{j}
$$

We conclude the $\alpha \leq r(A) \quad$ (by the theorem 5.1.8)

If $\alpha x<A x$, then there is some $a^{\prime}>a$ such that $a^{\prime} x \leq A x$.

In this event, $r(A)>a^{\prime}>a$.

If $A x \leq \beta x$, then $\beta x-A x \geq 0$. So

$$
\beta x_{i}-\sum_{j=1}^{n} a_{i j} x_{j} \geq 0 \text { for all } i=1,2, \ldots, n
$$

Then

$$
\beta \geq \max x_{i}^{-1} \sum_{j=1}^{n} a_{i j} x_{j} \geq r(A) \text { (by theorem } 5.1 .8 \text { ) }
$$

If $A x<\beta x$, then there is some $\beta^{\prime}<\beta$ such that $A x \leq \beta^{\prime} x$, so we have that

$$
r(A) \leq \beta^{\prime}<\beta
$$

Corollary 5.1.10: Let $A \in M_{n}$, and suppose that $A$ in nonngative. If $A$ has a postive eigenvector, then the corresponding eingenvalue is $r(A)$, that is,

if $A x=\lambda x, x>0$ and $A \geq 0$, then $\lambda=r(A)$.

Proof: If $x>0$ and $A x=\lambda x$, then $\lambda \geq 0$ and $\lambda x \leq A x \leq$ $\lambda x$, But then

$$
\lambda \leq r(A) \leq \lambda(\text { by corollary } 5.1 .9)
$$

So,

$r(A)=\lambda$

\subsection{Positive matrices}

Lemma 5.2.1: Let $A \in M_{n}$ and suppose that $A>0, A x=$ $\lambda x, x \neq 0$ and $|\lambda|=r(A)$

Then

$$
A|x|=r(A)|x| \text { and }|x|>0 .
$$

Proof:

Since $A x=\lambda x$ and $|\lambda|=r(A)$, we have

$$
r(A)|x|=|\lambda||x|=|\lambda x|=|A x| \leq|A||x|=A|x|
$$

So that

$$
y=A|x|-r(A)|x| \geq 0
$$

Since $|x| \geq 0$ And $|x| \neq 0$ we have

$$
A|x|>0 \text { by property(7)Theorem 3.2.13 }
$$

And we have that

$r(A)>0$ by corollary 5.1 .7 
So if $y=0$, we have

$$
A|x|=r(A)|x|
$$

If $y \neq 0$, set $z=A|x|>0$, Since $y \geq 0, y \neq 0$ and $A>0$, we have

$$
\text { Ay }>0 \text { (by property (7)of theorem 3.2.13) }
$$

$$
=A z-r(A) z>0 \text {, and so } A z
$$$$
>r(A) z \text {. But then by corollary 5.1.9 }
$$

We have that

$r(A)>r(A)$. So, $y=0$, and this completes the proof.

Theorem 5.2.2: Let $A \in M_{n}$, and suppose that $A>0$. Then $r(A)>0, r(A)$ is an eigenvalue of $A$, and there is a positive vector $x$ such that $A x=r(A) x$.

\section{Proof:}

There is an eigenvalue $\lambda$ with $|\lambda|=r(A)>0$ and an associated eigenvector $x \neq 0$ by the Lemma (5.2.1), the required vector is $|x|$.

Lemma 5.2.3: Let $A \in M_{n}$, and suppose that $A>0, A x=$ $\lambda x, x \neq 0$ and $|\lambda|=r(A)$. Then for some real number $\theta$

$$
e^{-i \theta} x=|x|>0
$$

Theorem 5.2.4:Let $A \in M_{n}$ and suppose $\mathrm{A}$ is a positive. Then $|\lambda|<r(A)$ For every eigenvalue $\lambda \neq r(A)$.

\section{Proof:}

For every eigenvalue $\lambda$ of, $|\lambda| \leq r(A)$.Suppose that $|\lambda|=r(A)$, and $A x=\lambda x, x \neq 0$. Then

$$
w=e^{-i \theta} x>0 \text { for some real number } \theta
$$

And

$$
A w=\lambda w
$$

But by corollary 5.1.10 we have $\lambda=r(A)$

Theorem 5.2.5: Let $A \in M_{n}$ and suppose that $A>0$ and that $w$ and $z$ are nonzero vectors such that $A w=r(A) w$ and $A z=$ $r(A) z$. Then there exists some $\alpha \in C$ such that $w=\alpha z$.

Proof:

Since $A>0$, and $w, z \neq 0$, there exist real number $\theta_{1}, \theta_{2}$ such that $\mathrm{p}=e^{-i \theta_{1}} z>0$ and

$\mathrm{q}=e^{-i \theta_{2}} w>0$. Set $\beta=\min _{1 \leq i \leq n} q_{i} p_{i}^{-1}$ and define $\mathrm{v}=-\beta p$

It follows that $v \geq 0$. For if $v<0$, then $q-\beta p<0$, iff $e^{-i \theta_{2}} w<\beta e^{-i \theta_{2}} z$, iff $e^{-i\left(\theta_{2}-\theta_{1}\right)} \frac{w}{z}<\beta$. This is a contradiction with $=\min _{1 \leq i \leq n} q_{i} p_{i}^{-1}$. So, $v \geq 0$ and at least one coordinate of $v$ is 0 . So $v$ is not a positive vector, But

$$
A v=A q-\beta A p=r(A) q-\beta r(A) p=r(A) v
$$

So if $v \neq 0$, then

$$
A v>0 \text { ( by theorem 3.2.13) }
$$

But since $r(A) v=A v>0$ and $r(A)>0$, we have

$$
v=r(A)^{-1} A v>0 \text { (this is not true) }
$$

So, $\mathrm{v}=0$ and hence $q=\beta p$ and $=\beta e^{i\left(\theta_{2}-\theta_{1}\right)} z$. Set $\alpha=$ $\beta e^{i\left(\theta_{2}-\theta_{1}\right)}$, then $W=\alpha z$.

Now we can define the Perron vector of $\mathrm{A} \in M_{n}$ as follow: If

$\in M_{n}$ and $A>$

0 , then there exists a unique vector $x$ such that $A x=r(A) x$,

$x>0$,

and $\sum_{i=1}^{n} x_{i}=$

1.This special eigenvector $x$ is called the perron vector for $A>$ 0 , and The associated eigenvalue $r(A)$ is called the Perron root of

A. Since $A>0$ iff $A^{t}>0$, and since $r(A)=r\left(A^{t}\right)$ it is clear that if $A>0$, then in addition to Perron eigenpair $(r(A), x)$ for $A$ there is a corresponding Perroneigenpair $(r(A), y)$ for $A^{t}$.

The vector $y^{t}>0$ is called the left hand Perron vector for $A$.

Remark 5.2.6:If $A \in M_{n}$ and $A>0$, and if there is some $z \in C^{n}$ such that $z \geq 0, z \neq 0$, and $A z=\lambda z$ then $z$ is a multiple of Perron vector of $A$ and that $\lambda=r(A)$.

Lemma 5.2.7: Let $\in M_{n}$, be given, let $\lambda \in C$ be given, and suppose $\mathrm{x}$ and $\mathrm{y}$ are vectors such that:

1) $A x=\lambda x$

2) $A^{t} y=\lambda y$, and

3) $x^{t} y=1$

Define $L=x y^{t}$, Then
a) $\quad L x=x$ and $y^{t} L=y^{t}$
b) $L^{m}=L$ for all $m=1,2, \ldots$
c) $A^{m} L=L A^{m}=\lambda^{m} L$ for all $m=1,2, \ldots$

d) $L(A-\lambda L)=0$

e) $(A-\lambda L)^{m}=A^{m}-\lambda^{m} L$ for all $m=1,2, \ldots$

f) Every nonzero eigenvalue of $A-\lambda L$ is also an eigenvalue of $A$.

If, in addition, we assume that

1) $\lambda \neq 0$

2) $\lambda$ is an eigenvalue of $A$ with geometric multiplicity 1 , then we also have that

a) $\lambda$ is not an eigenvalue of $A-\lambda L$

Finally, if we assume that

3) $|\lambda|=r(A)>0$

4) $\lambda$ is the only eigenvalue of $A$ with modulus $r(A)$ and if we order the eigenvalues of $A$ as $\quad\left|\lambda_{1}\right| \leq\left|\lambda_{2}\right| \leq \ldots \leq\left|\lambda_{n-1}\right| \leq\left|\lambda_{n}\right|=|\lambda|=$ $r(A)$, then

a) $\quad r(A-\lambda L) \leq\left|\lambda_{n-1}\right|<r(A)$

b) $\left(\lambda^{-1} A\right)^{m}=L+\left(\lambda^{-1} A-L\right)^{m} \rightarrow L$ as $m \rightarrow \infty$

For every $k$ such that $\left(\frac{\left|\lambda_{n-1}\right|}{r(A)}\right)<k<1$ there exists some $c=$ $c(k, A)$, such that

$$
\left\|\left(\lambda^{-1} A\right)^{m}-L\right\|_{\infty}<c k^{m}, \text { for all } m=1,2, \ldots
$$




\section{Proof:}

The proofs of (a) - (f) are obvious. We notice, that $\mathrm{x}$ and $\mathrm{y}$ are nonzero vectors.

Proof of $(\mathrm{g})$ :

Suppose $\lambda$ is an eigenvalue of $A-\lambda L$, then for some eigenvector $w \neq 0,(A-\lambda L) w=\lambda w$.

But by (5) we must then conclude that $w=\alpha x$ for some $\alpha \neq 0$. Now,

$$
\begin{aligned}
\lambda w=(A & -\lambda L) w=(A-\lambda L) \alpha x \\
& =A \alpha x-\lambda L \alpha \\
& =\alpha A x-\lambda \alpha L x
\end{aligned}
$$

$=\alpha \lambda x-\lambda \alpha x \quad($ by $(\mathrm{a}))$

$$
=0
$$

Which is impossible since $\lambda \neq 0$ and $w \neq 0$

\section{Proof of (h):}

By (f), (g), if we order the eigenvalue of $A$ as

$\left|\lambda_{1}\right| \leq\left|\lambda_{2}\right| \leq \ldots \leq\left|\lambda_{n-1}\right| \leq\left|\lambda_{n}\right|=|\lambda|=r(A)$,Then we have

$$
r(A-\lambda L) \leq\left|\lambda_{n-1}\right|<\left|\lambda_{n}\right|=|\lambda|=r(A)
$$

\section{Proof of (i):}

By (e), we have that

$$
(A-\lambda L)^{m}=A^{m}-\lambda^{m} L \text { for all } m=1,2, \ldots .
$$

So,

$$
\lambda^{m}\left(\lambda^{-1} A-L\right)^{m}=\lambda^{m}\left(\left(\lambda^{-1} A\right)^{m}-L\right)
$$

Then,

$$
\left(\lambda^{-1} A\right)^{m}=L+\left(\lambda^{-1} A-L\right)^{m}
$$

Notice That

$$
r\left(\lambda^{-1} A-L\right)=\frac{r(A-\lambda L)}{r(A)} \leq \frac{\left|\lambda_{n-1}\right|}{r(A)}<\frac{r(A)}{r(A)}=1
$$

So, by theorem 4.1.8, $\lim _{m \rightarrow \infty}\left(\lambda^{-1} A-L\right)^{m}=0$

Then,

$$
\left(\lambda^{-1} A\right)^{m} \rightarrow \text { When } m \rightarrow \infty
$$

Proof of (j):

Applying (corollary 4.1.9) to the matrix $\lambda^{-1} A-L$ as follows. Let $\varepsilon>0$. Then there is a constant $c>0$ such that

$$
\begin{aligned}
&\left|\left(\lambda^{-1} A-L\right)_{i, j}^{m}\right| \leq c\left(r\left(\lambda^{-1} A-L\right)+\varepsilon\right)^{m} \\
& \leq c\left(\frac{\left|\lambda_{n-1}\right|}{r(A)}+\varepsilon\right)^{m} \\
&<c(k+\varepsilon)^{m}
\end{aligned}
$$

Since $\varepsilon>0$ is arbitrary, it follows that

$$
\left\|\left(\lambda^{-1} A L\right)^{m}\right\|_{\infty}<c k^{m} \text { for all } m=1,2, \ldots
$$

Theorem 5.2.8: Let $A \in M_{n}$ and suppose that $\mathrm{A}>0$. Then

$$
\lim _{m \rightarrow \infty}\left(r(A)^{-1} A\right)^{m}=L,
$$

Where

$$
\begin{gathered}
L=x y^{t}, A x=\underset{=1}{r}(A) x, A^{t} y=r(A) y, x>0, y>0 \text {, and } x^{t} y \\
=1
\end{gathered}
$$

Proof:

The assumptions (1)-(7) of lemma (5.2.7) are satisfied with $\lambda=$ $r(A)$, x the Perron vector of $A$, and $y=\left(x^{t} z\right)^{-1} z$, where $z$ is the Perron vector of $A^{t}$. The conclusion follows from (i).

Theorem 5.2.9: (Perron's theorem ). If $A \in M_{n}$ and $A>0$, then

a) $r(A)>0$

b) $\quad r(A)$ is an eigenvalue of $A$

c) There is an $x \in C^{n}$ with $x>0$ and $A x=r(A) x$.

d) $|\lambda|<r(A)$ for every eigenvalue $\lambda \neq r(A)$

e) $\left(r(A)^{-1} A\right)^{m} \rightarrow L$ as $m \rightarrow \infty$ where $L=x y^{t}, A x=$ $r(A) x, A^{t} y=r(A) y, x>0$,

$$
y>0, \text { and } x^{t} y=1
$$

Corollary 5.2.10: If $A>0$ and if $x$ is the Perron vector of $A$, then

$$
r(A)=\sum_{i, j=1}^{n} a_{i j} x_{j}
$$

Proof:

By Perron's Theorem $A x=r(A) x$. So

$$
\sum_{j=1}^{n} a_{i j} x_{j}=r(A) x_{i} \text { for all } i=1,2, \ldots, n
$$

Thus

$$
\sum_{i, j=1}^{n} a_{i j} x_{j}=\sum_{i=1}^{n} r(A) x_{i}=r(A) \sum_{i=1}^{n} x_{i}=r(A)
$$

Theorem 5.2.11: If $A \in M_{n}$ and $A \geq 0$, then $r(A)$ is an eigenvalue of $A$ and there is a nonnegative vector $x \geq 0, x \neq 0$, such that $A x=r(A) x$.

Proof:

For any $\varepsilon>0$, define $\mathrm{A}(\varepsilon)=\left|a_{y}+\varepsilon\right|>0$. Denote by $x(\varepsilon)$ the Perron vector of $A(\varepsilon)$, so $x(\varepsilon)>0$ and

$$
\begin{array}{r}
\sum_{i=1}^{n} x_{i}(\varepsilon)=1 . \text { Since the set of vectors }(x(\varepsilon): \varepsilon \\
>0) \text { is contained in the set }
\end{array}
$$

$\left\{x: x \in C^{n},\|x\|_{1} \leq 1\right\}$, there is a monotone decreasing sequence $\varepsilon_{1}, \varepsilon_{2}, \ldots$ with

$$
\lim _{k \rightarrow \infty} \varepsilon_{k}=0 \text { such that } \lim _{k \rightarrow \infty}\left(\varepsilon_{k}\right)=x \text { exisit. }
$$

Since $x\left(\varepsilon_{k}\right)>0$ for all $k=1,2, \ldots$, it must be that $x=$ $\lim _{k \rightarrow \infty} \varepsilon_{\mathrm{k}} \geq 0$ and $x \neq 0$, because 


$$
\sum_{i=1}^{n} x_{i}=\lim _{k \rightarrow \infty} \sum_{i=1}^{n} x_{i}\left(\varepsilon_{k}\right)=1
$$

Since there is a monotone decreasing sequence $\varepsilon_{1}, \varepsilon_{2}, \ldots$ with $\lim _{k \rightarrow \infty} \varepsilon_{k}=0$, we have

$r\left(A\left(\varepsilon_{k}\right)\right) \geq r\left(A\left(\varepsilon_{k+1}\right)\right) \geq \cdots \geq r(A) \quad($ by Theorem 5.1.1)

For all $\mathrm{k}=1,2 \ldots$ So the sequence of real number $\left\{r\left(A\left(\varepsilon_{k}\right)\right)\right\}$ for $\mathrm{k}=1,2 \ldots$ is a monotone decreasing sequence. Thus, $\mathrm{r}=\lim _{k \rightarrow \infty} r\left(A\left(\varepsilon_{k}\right)\right)$, exists and $r>r(A)$.

But from the fact that

$$
\begin{gathered}
A x=\lim _{k \rightarrow \infty} A\left(\varepsilon_{k}\right) x\left(\varepsilon_{k}\right)=\lim _{k \rightarrow \infty} r\left(A\left(\varepsilon_{k}\right)\right) x\left(\varepsilon_{k}\right) \\
\lim _{k \rightarrow \infty} r\left(A\left(\varepsilon_{k}\right)\right) \lim _{k \rightarrow \infty} x\left(\varepsilon_{k}\right)=r x
\end{gathered}
$$

And the fact that $x \neq 0$, we deduce that $r$ is an eigenvalue of $A$. But then $r \leq r(A)$, so it must be that $r=r(A)$.

Theorem 5.2.12: Let $A \in M_{n}, A \geq 0, x \in C^{n}, x \geq 0$, and $x \neq$ 0.If $A x \geq \alpha x$ for some real number $\alpha$ then $r(A) \geq \alpha$.

Proof:

Let $A=\left\lfloor a_{i j}\right\rfloor$, Let $\varepsilon>0$, and define $A(\varepsilon)=\left\lfloor a_{i j}+\varepsilon\right\rfloor$. Then $A(\varepsilon)>0$, so $A(\varepsilon)$ has a positive left Perron vector $\mathrm{y}(\varepsilon)$, that is

$y^{t}(\varepsilon) A(\varepsilon)=r(A(\varepsilon)) y^{t}(\varepsilon)$.

Since $A x \geq a x$, we have that

$$
A x-a x \geq 0
$$

So

$$
A(\varepsilon) x-\alpha x \geq A x-\alpha x \geq 0 .
$$

Hence

$$
y^{t}(\varepsilon)[A(\varepsilon) x-\alpha x]=[r(A(\varepsilon))-\alpha] y^{t}(\varepsilon) x \geq 0
$$

Since $y^{t}(\varepsilon) x>0$, we have:

$$
r(A(\varepsilon))-\alpha \geq 0 \text { for all } \varepsilon>0 .
$$

But $r(A(\varepsilon)) \rightarrow r(A)$ as $\varepsilon \rightarrow 0$, so we conclude that $r(A) \geq \alpha$

\subsection{Upper bounds of the perron roots of nonnegative matrices}

For Any nonnegative matrix $A=\left[a_{i j}\right] \in M_{n}$, the numbers $T(A), N(A), \tau_{k}, \varphi_{k}$ are defined by:

$$
\begin{gathered}
T(A)=\sum_{i, j=1}^{n} a_{i j}, N(A)=n \max _{i, j} a_{i j}, \tau_{k}=\left(T\left(A^{2^{k}}\right)\right)^{2^{-k}}, \text { and } \varphi_{k} \\
=\left(N\left(A^{2^{k}}\right)\right)^{2^{-k}}
\end{gathered}
$$

Lemma 5.3.1: If $A=\left\lfloor a_{\mathrm{ij}}\right\rfloor \in M_{n}$ is nonnegative matrix, then $r(A) \leq T(A)$.

\section{Proof:}

If $A \geq 0$, then by Theorem 5.2.11, there is a nonnegative vector $x \geq 0$, such that $A x=r(A) x$.
Let $x$ be the Perron vector of $A$. Then

$$
\begin{gathered}
r(A) \sum_{i=1}^{n} x_{i}=\sum_{i=1}^{n} \sum_{j=1}^{n} a_{i j} x_{j} \leq\left(\sum_{i, j=1}^{n} a_{i j}\right)\left(\sum_{j=1}^{n} x_{j}\right)=\sum_{i, j=1}^{n} a_{i j} \\
=T(A) .
\end{gathered}
$$

Lemma 5.3.2: If $A=\left\lfloor a_{\mathrm{ij}}\right\rfloor \in M_{n}$ is nonnegative matrix, then $r(A) \leq N(A)$.

Proof: If $A \geq 0$, then by Theorem 5.2.11, there is a nonnegativevector $x \geq 0, x \neq 0$, and $x=\left(x_{1}, x_{2}, \ldots, x_{n}\right)^{t}$ corresponding to $r(A)$. Let $x_{\mu}=\max _{1 \leq i \leq n}\left\{x_{i}\right\}$. Then

$r(A) x_{i}=\sum_{j=1}^{n} a_{i j} x_{j}$

And Hence

$$
r(A) x_{\mu}=\max _{1 \leq i \leq n}\left(\sum_{j=1}^{n} a_{i j} x_{j}\right) \leq n \max _{i, j}\left(a_{i j} \mathrm{x}_{j}\right) .
$$

Since $\frac{x_{j}}{x_{\mu}} \leq 1$ for $1 \leq j \leq n$, we have

$$
r(A) \leq n \max _{i, j}\left(a_{i j} \frac{x_{j}}{x_{\mu}}\right) \leq n \max _{i, j}\left(a_{i j}\right)=N(A) .
$$

Theorem 5.3.3: If $A=\left\lfloor a_{\mathrm{ij}}\right\rfloor \in M_{n}$ is a nonnegative matrix, then for $k=1,2, \ldots$
1) $r(A) \leq \tau_{k}$
2) $r(A) \leq \tau_{k} \leq \tau_{k-1} \leq \cdots \leq \tau_{1} \leq \tau_{0}$
3) $\lim _{k \rightarrow \infty} \tau_{k}=r(A)$

Proof:

1) We first note that the inequality (1) implies

$(r(A))^{2^{k}}=r\left(A^{2^{k}}\right) \leq T\left(A^{2^{k}}\right) \quad($ By Lemma 5.3.1)

And hence

$$
r(A)=\left(r(A)^{2^{k}}\right)^{2^{-k}} \leq\left(T(A)^{2^{k}}\right)^{2^{-k}}=\tau_{k}
$$

2) Let $t=t_{0}=\mathrm{T}(A)$, and notice that

$$
\begin{gathered}
T\left(A^{2}\right)=\sum_{i, j=1}^{n}\left(\sum_{k=1}^{n}\left(a_{i k} a_{k j}\right)\right)=\sum_{i, j, k=1}^{n}\left(a_{i k} a_{k j}\right) \\
\leq \sum_{i, j, k, m=1}^{n}\left(a_{i k} a_{m j}\right)
\end{gathered}
$$

$=T(A) T(A)=(T(A))^{2}=\tau^{2}$, so

$\tau_{1}=\left(T\left(A^{2}\right)\right)^{\frac{1}{2}} \leq\left(\tau^{2}\right)^{\frac{1}{2}}=\tau$

$\tau_{2}=\left(T\left(A^{4}\right)\right)^{\frac{1}{4}}=\left(T\left(A^{2} A^{2}\right)\right)^{\frac{1}{4}} \leq\left(T\left(A^{2}\right)\right)^{\frac{1}{4}}\left(T\left(A^{2}\right)\right)^{\frac{1}{4}}=\left(\tau_{1}\right)^{\frac{1}{2}}\left(\tau_{1}\right)^{\frac{1}{2}}$

$$
=\tau_{1}
$$

And so on.

3) Since $T(A)$ is a matrix norm, Corollary 4.1.9 implies that

$$
\lim _{k \rightarrow \infty}\left(T(A)^{2^{k}}\right)^{2^{-k}}=r(A) .
$$


Theorem 5.3.4: If $\mathrm{A}=\left\lfloor a_{\mathrm{ij}}\right\rfloor \in M_{n}$ is a nonnegative matrix, then for $k=1,2, \ldots$

1) $r(A) \leq \varphi_{k}$

2) $r(A) \leq \varphi_{k} \leq \varphi_{k-1} \leq \cdots \leq \varphi_{1} \leq \varphi_{0}$

3) $\lim _{k \rightarrow \infty}\left(N(A)^{2^{k}}\right)^{2^{-k}}=r(A)$

Proof:

1) It follows from the Lemma 5.3.2 that

$$
r(A)^{2^{k}}=r\left(A^{2^{k}}\right) \leq N\left(A^{2^{k}}\right)
$$

Which implies that

$$
r(A)=r\left(A^{2^{k}}\right)^{2^{-k}} \leq\left(N(A)^{2^{k}}\right)^{2^{-k}}=\varphi_{k}
$$

2) Let $\varphi=\varphi_{0}=N(A)$, notice that

$$
\begin{gathered}
N\left(A^{2}\right)=n \max _{i, j}\left(\sum_{k=1}^{n} a_{i k} a_{k j}\right) \leq n \max _{i, j}\left(n \max _{i, j, k} a_{i k} a_{k j}\right) \\
=\left(n \max _{i, k} a_{i k}\right)\left(n \max _{k, j} a_{k j}\right) \\
=N(A) N(A)=\varphi^{2}, \text { so } \\
\varphi_{1}=N\left(A^{2}\right)^{\frac{1}{2}} \leq\left(\varphi^{2}\right)^{\frac{1}{2}}, s o \\
\varphi_{2}=N\left(A^{4}\right)^{\frac{1}{4}} \leq N\left(A^{2}\right)^{\frac{1}{4}} N\left(A^{2}\right)^{\frac{1}{4}}=\varphi_{1}^{\frac{1}{2}} \varphi_{1}^{\frac{1}{2}}=\varphi_{1}, \\
\varphi_{3}=N\left(A^{8}\right)^{\frac{1}{8}} \leq N\left(A^{4}\right)^{\frac{1}{8}} N\left(A^{4}\right)^{\frac{1}{8}}=\varphi_{2}^{\frac{1}{2}} \varphi_{2}^{\frac{1}{2}}=\varphi_{2}
\end{gathered}
$$

And so on.

We conclude this study with two examples illustrating the conclusions of both theorem 5.3.3 and theorem 5.3.4

For $A=\left[\begin{array}{lll}2 & 1 & 3 \\ 3 & 2 & 1 \\ 5 & 4 & 2\end{array}\right], r(A)=7.4354$

The first four terms of the sequences for $\mathrm{A}$,

$$
\begin{aligned}
\tau=\tau_{0}=23, \tau_{1}= & 12.9615, \tau_{2}=9.8236, \tau_{3}=8.5465, \tau_{4} \\
& =7.9716 \\
\varphi=\varphi_{0}=15, \varphi_{1}= & 9.7980, \varphi_{2}=8.5687, \varphi_{3}=7.9820, \varphi_{4} \\
= & 7.7038
\end{aligned}
$$

For $B=\left[\begin{array}{lll}2 & 0 & 1 \\ 0 & 1 & 0 \\ 3 & 6 & 1\end{array}\right], r(B)=3.3028$

The first four terms of the sequences for B,

$$
\begin{aligned}
& \tau=\tau_{0}=14, \tau_{1}=6.4807, \tau_{2}=4.6058, \tau_{3}=3.8992, \tau_{4} \\
& =3.5886 \\
& \varphi=\varphi_{0}=18, \varphi_{1}=6, \varphi_{2}=4.3004, \varphi_{3}=3.7606, \varphi_{4} \\
& =3.5242
\end{aligned}
$$

\section{Conclusion}

This study has obtained many matrix inequalities involving matrix norm and spectral radius of nonnegative and positive matrices using the concepts of maps on matrix space. And it has investigated the lower and upper bounds of spectral radius for nonnegative and positive matrices.

Finally, after examining the results for bounding column (row) sums with spectral radius of nonnegative and positive matrices, the study has been well proved to upper bounds of the Perron roots of nonnegative and positive matrices.

\section{References}

[1] Barraa, M. and Boumazgour, M. (2001). Inner derivations and norm equality,Proc. Amer. Math. Soc. 130:471476.https://doi.org/10.1090/S0002-9939-01-06053-1.

[2] Bhatia, R. (1997). Matrix Analysis, Springer-Verlag, New York.https://doi.org/10.1007/978-1-4612-0653-8.

[3] Cheng, G-H., Cheng, X-Y., Huang, T-Z., and Tam, T-Y. (2005). Some Bounds for the spectralradius of the Hadamrad product of matrices, Applied Math. E-Notes. 5: 202-209

[4] Fujii, M. and Kubo, F. (1993). Buzano's inequality and bounds for roots of algebraic equations, proc. Amer. math. soc. 117:359-361.

[5] Halmos, P.R. (1982). A Hillbert Space Problem Book, 2nd ed. Springer-Verlag, New York. https://doi.org/10.1007/978-1-46849330-6.

[6] Horn, R. A. and Johnson, C. R. (1985). Matrix Analysis, Cambridge University Press, Cambridge.https://doi.org/10.1017/CBO9780511810817.

[7] Horn, R. A. and Johnson, C.R. (1991). Topics in Matrix Analysis, Cambridge University Press, Cambridge. https://doi.org/10.1017/CBO9780511840371.

[8] Hou, I.C. and Du, H. K. (1995). Norm inequalities of positive operator matrices, Integral Equations Operator Theory 22:281294.https://doi.org/10.1007/BF01378777.

[9] Kittaneh, F. (2002). Norm inequalities for sums of positive operator, J. Operator Theory 48:95-103.

[10] Kittaneh, F.2003. A numerical radius inequality and an estimate for the numerical radius of the Forbenius companion matrix, StudiaMath.158:11-17. https://doi.org/10.4064/sm158-1-2.

[11] Kittaneh, F. (2005). Spectral radius inequalities for Hilbert Space operato, Porc. Amer. math. Soc.143:385-390

[12] Kittaneh, F. (2005). Numerical radius inequalities for Hilbert Space operator,Studia Math., 168:73-80.https://doi.org/10.4064/sm168-15.

[13] Omladic, M., Radjavi, H., Rosenthal, P., and Sourour, A. (2001) Inequalities for the products of spectural radii, Proc. Amer. Math. Soc. 129:2239-2243.https://doi.org/10.1090/S0002-9939-01-055009.

[14] Tasci, D. and Celik, H. A. On the upper bounds of the Perron roots of nonnegative matrices, Selcuk University, Konya, Turkey. Math. to appear.

[15] Zhang, F. (1999). Matrix Theory, Springer- Verlag, New York.https://doi.org/10.1007/978-1-4757-5797-2. 